\title{
Microcephaly-cardiomyopathy syndrome
}

INSERM

\section{Source}

INSERM. (1999). Orphanet: an online rare disease and orphan drug data base.

Microcephaly-cardiomyopathy syndrome. ORPHA:2515

Microcephaly-cardiomyopathy syndrome is characterised by severe intellectual deficit, microcephaly and dilated cardiomyopathy. Hand and foot anomalies have also been reported. The syndrome has been described in three individuals. Transmission is autosomal recessive. 